2 Received Date : 25-Feb-2016

3 Revised Date : 21-Jul-2016

4 Accepted Date : 01-Sep-2016

5 Article type : Original

6

7

8

Title page

9 Title: Is there a march from early food sensitization to later childhood allergic airway

disease? Results from two prospective birth cohort studies

Authors

13 Shatha A. Alduraywish ${ }^{1,2}$, Marie Standl ${ }^{3}$, Caroline J. Lodge ${ }^{1}$, Michael J. Abramson ${ }^{4}$, 14 Katrina J. Allen ${ }^{5}$, Bircan Erbas ${ }^{6}$, Andrea v. Berg ${ }^{7}$, Joachim Heinrich ${ }^{3,8}$, Adrian J Lowe* ${ }^{1,5}$, 15 Shyamali C Dharmage* ${ }^{1,5}$

16 From

$17{ }^{1}$ Allergy and Lung Health Unit, Centre for Epidemiology and Biostatistics, Melbourne 18 School of Population and Global Health, University of Melbourne, Melbourne-Australia; ${ }^{2}$ 19 the Department of Family and Community Medicine, King Saud University, Riyadh-Saudi 20 Arabia; ${ }^{3}$ Institute of Epidemiology I, Helmholtz Zentrum München - German Research 21 Center for Environmental Health, Munich-Germany; ${ }^{4}$ School of Public Health \& Preventive 22 Medicine, Monash University, Melbourne-Australia; ${ }^{5}$ Murdoch Children's Research 23 Institute, University of Melbourne Department of Paediatrics, Royal Childrens' Hospital, 24 Melbourne-Australia; ${ }^{6}$ School of Psychology and Public Health, La Trobe University, 25 Melbourne-Australia; ${ }^{7}$ Marien-Hospital Wesel, Research Institute, Department of 26 Pediatrics, Wesel-Germany; ${ }^{8}$ Institute and Outpatient Clinic for Occupational, Social and 27 Environmental Medicine Unit, Paediatric Environmental Epidemiology, WHO Collaboration 28 Centre for Occupational Health University Hospital Munich, Ziemssenstr. 180336 München, 29 Munich-Germany.

$30 *$ equal senior authors

This is the author manuscript accepted for publication and has undergone full peer review but has not been through the copyediting, typesetting, pagination and proofreading process, which may lead to differences between this version and the Version of Record. Please cite this article as doi: $10.1111 /$ pai.12651

This article is protected by copyright. All rights reserved 
32 Running title: Food sensitization and allergic airway disease

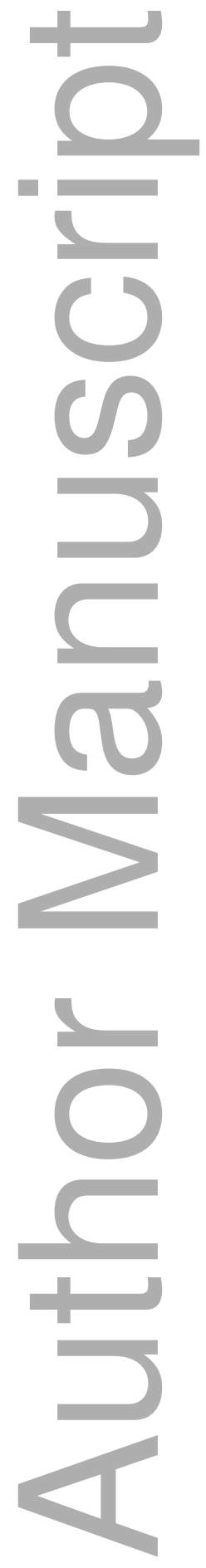

This article is protected by copyright. All rights reserved 
34 Corresponding author

35 Professor Shyamali C Dharmage MBBS, MSc, MD, PhD

36 Allergy and Lung Health Unit

37 Centre for Epidemiology and Biostatistics

38 Melbourne School of Population and Global Health, The University of Melbourne

39207 Bouverie Street, Carlton, Vic 3010

40 Tel +61383440737 Fax: +61393495815

41 E mail s.dharmage@unimelb.edu.au

Word count:-Abstract (264), Text (3379)

44 Number of tables and figures: Tables (3), No figures

45 Material in the electronic repository: 1 table

46 Abstract page

47 Alduraywish S, Standl M, Lodge C, Abramson M, Allen K, Erbas B, Berg A,

48 Heinrich A, Lowe A, Dharmage S C

49 Title: Is there a march from early food sensitization to later childhood allergic airway

50 disease? Results from two prospective birth cohort studies

51 Title of Journal: Pediatr Allergy Immunol

52 Abstract:

53 Background: The march from early aeroallergen sensitization to subsequent

54 respiratory allergy is well established, but it is unclear if early life food sensitization

55 precedes and further increases risk of allergic airway disease.

56 Objective: To assess the association between food sensitization in the first 2 years of

57 life and subsequent asthma and allergic rhinitis by age 10-12 years.

58 Methods: We used data from two independent cohorts: the high-risk MACS ( $\mathrm{n}=620)$

59 and the population-based LISAplus $(n=3094)$. Food sensitization was assessed at 6 , 
6012 and 24 months in MACS and 24 months in LISAplus. Multiple logistic regressions

61 were used to estimate associations between sensitization to food only, aeroallergen

62 only or both and allergic airway disease.

63 Results: When compared to non-sensitized children, sensitization to food only at 12

64 months in MACS and 24 months in LISAplus was associated with increased risk of 65 current asthma $(\mathrm{aOR}=2.2 ; 95 \% \mathrm{CI} 1.1,4.6$ in $\mathrm{MACS}$ and $\mathrm{aOR}=4.9 ; 2.4,10.1$ in

66 LISAplus). Similar results were seen for allergic rhinitis. Additionally, co-

67 sensitization to food and aeroallergen in both cohorts at any tested point was a

68 stronger predictor of asthma (at 24 months, aOR=8.3; 3.7, 18.8 in MACS and

$69 \mathrm{aOR}=14.4 ; 5.0,41.6$ in LISAplus) and allergic rhinitis (at 24 months,

$70 \mathrm{aOR}=3 \cdot 9 ; 1 \cdot 9,8.1$ in MACS and $\mathrm{aOR}=7 \cdot 6 ; 3 \cdot 0,19 \cdot 6$ in LISAplus).

71 Conclusions: In both cohorts, food sensitization (with or without aeroallergen

72 sensitization) in the first two years of life increased the risk of subsequent asthma and

73 allergic rhinitis. These findings support the role of early life food sensitization in the

74 atopic march and suggest trials to prevent early onset have the potential to reduce the 75 development of allergic airways disease.

Key words: Allergic rhinitis, Asthma, Atopy, Food Sensitization

Abbreviation:

80

aOR: Adjusted odd ratio

LISAplus: Influence of Life-style related factors on the development of the Immune System and Allergies in East and West Germany study

83 MACS: Melbourne Atopic Cohort Study

84 S-IgE: serum specific immunoglobulin E

87 Over the past 50 years there has been a global epidemic of asthma, eczema and allergic rhinitis, especially in developed countries (1). Over the last 20 years, evidence suggests a second wave of the allergy epidemic with an increase in the prevalence of food allergies $(2,3)$. These allergic disorders pose a substantial health

91 burden on affected individuals, their families and healthcare resources (4-6). Given

92 the overlap between allergic disorders, the link between them has been a major 93 research focus. 
94 The "atopic march" refers to progression of allergic phenotype from early life eczema into later asthma and allergic rhinitis, which has been supported by many longitudinal and cross-sectional studies (7-9). There is increasing interest in these longitudinal relationships because the information could contribute in identifying early interventions and reduce burden of these disorders. Eczema is commonly associated with atopic sensitization, as assessed by either skin prick test (SPT) or serum specific $\operatorname{IgE}$ in vitro (s-IgE) (10). Previous studies suggest that eczema along with atopy is considered as a major risk factor for progression in the atopic march (11). Data from Melbourne Atopic Cohort Study (MACS) showed that children with atopic eczema in the first two years of life had a greater risk of asthma and allergic rhinitis at 6 and 7 years when compared with children with non-atopic eczema (9). Although several epidemiological studies have shown that early aeroallergen sensitization is related to increased risk of allergic diseases in children (12-15) and adults (16), the role of food sensitization is less clear. Considering that food sensitization tends to develop earlier than aeroallergen sensitization, measuring food sensitization in the early years of life may allow earlier prediction of childhood and

111 in early life.

112 Food sensitization has been hypothesised to be related to development of other 113 allergic diseases including asthma, allergic rhinitis $(8,17,18)$ and food allergy (19).

114 Although a number of epidemiological studies have assessed the association between

115 food sensitization and subsequent asthma and/or allergic rhinitis up to seven years

$116(12,20,21)$, only a few cohorts have assessed these associations beyond this age (22-

117 24). However, concomitant early life eczema and/or wheeze have not been considered 118 in most studies.

119 We conducted prospective analyses of two independent cohorts: the high-risk 120 Australian based MACS cohort and the population based Influence of Life-style 121 related factors on the development of the Immune System and Allergies in East and

122 West Germany plus the influence of traffic emissions and genetics (LISAplus) cohort.

123 We investigated the association between food sensitization, with or without

124 aeroallergen sensitization, at different time points in the first two years of life and risk

125 of allergic airway disease by age 10-12 years, whilst taking into account various

126 confounding factors. 


\section{Study populations}

130 MACS began as a randomized controlled trial investigating the effect of three

131 different infant formulas (cow's milk, partially hydrolysed whey and standard soy

132 formulas) introduced at the time of weaning on the occurrence of allergic diseases. A

133 total of 620 infants born between 1990 and 1994 were recruited from antenatal clinics

134 at the Mercy Maternity Hospital in Melbourne, Australia. Children were eligible if

135 they had a first degree relative with asthma, eczema, hay fever or food allergy.

136 Baseline information was collected during pregnancy.

137 Telephone-based interviews were conducted every 4 weeks until 15 months, at 18

138 months, 2 years then annually up to the age of 7 years, then at 12 and 18 years. At the

13912 year follow-up, the mean $( \pm \mathrm{SD})$ age of participants was $11.5 \pm 2$ years. The study

140 was approved by the Human Research Ethics committees of the Mercy Maternity

141 Hospital and University of Melbourne. Written informed consent was obtained from

142 all mothers and/or participants.

143 The baseline demographics of MACS participants have been published previously (9).

144 Briefly, parents of MACS children were mainly Australian born (83\% of fathers and

$14587 \%$ of mothers) and well educated (61\% of fathers and $59 \%$ of mother had higher

146 education).

147 Using data from randomized controlled trials to test additional hypotheses about the

148 association between non-randomized exposures and outcomes determined during

149 long-term follow-up is a well-established method. It is based on the testable

150 assumption that the randomized intervention does not influence the associations of

151 interest (25). A previous MACS publication showed that the randomization status

152 (infant formula allocation) was not associated with the outcome of interest (26),

153 therefore MACS continues as an observational study. Despite this, the effect of an

154 intervention formula (by intention to treat at baseline) was considered as a potential

155 confounder in all analyses.

157 LISAplus is a German population based birth cohort study that recruited 3094

158 neonates between 1997 and 1999 from the cities of Munich, Leipzig, Wesel and Bad

159 Honnef. Questionnaires were completed by parents at birth, 6 months, 1, 1.5, 2, 4, 6

160 and 10 years of age. Details of the study design have been described elsewhere (27).

161 The study was approval by the local Ethics Committees (Bavarian Board of 
162 Physicians, University of Leipzig, and Board of Physicians of North-Rhine-

163 Westphalia) and written parental consent was obtained.

164 The demographic characteristics for the initial LISAplus cohort have been described

165 in previous publications (27).

167 Sensitization assessment

168 In MACS, Skin Prick Tests (SPT) were performed at 6, 12 and 24 months, according 169 to a standard technique (28). Allergens tested included egg white, cow's milk,

170 peanut, house dust mite (Dermatophagoides pteronyssinus), rye grass (Lolium

171 perenne) and cat dander (Bayer, Spokane, WA, USA). A positive histamine control (1

$172 \mathrm{mg} / \mathrm{mL}$ ) was used. SPTs were read at 15-20 minutes. Wheal size was measured by

173 calculating the mean length of the longest and perpendicular wheal diameters (15).

174 Sensitization was defined as wheal size of $\geq 2 \mathrm{~mm}$ (29).

175 In LISAplus, blood samples were collected at the age of 2 years. Serum-specific IgE

176 antibodies (s-IgE) were measured using a mix of common food allergens (FX5: hen's

177 egg, cow's milk, peanut, wheat flour, soybean, and codfish). If the specific IgE

178 exceeded $0.35 \mathrm{kU}_{\mathrm{A}} / \mathrm{L}$, egg white, milk protein and peanuts protein were tested

179 separately. The inhalant allergens HX2 (mite), E1 (cat), MX1 (mold), RX1 (pollen)

180 were tested separately. Standardised methods with the CAP System FEIA (Pharmacia

181 Diagnostics, Freiburg, Germany) were used. Sensitization was defined as an IgE

182 antibody level $\geq 0.35 \mathrm{kU}_{\mathrm{A}} / \mathrm{L}$.

183

184 Outcome definitions

185 Allergic outcomes were defined by questionnaire responses at age 10 year follow-up

186 in LISAplus and at 12 year follow-up in MACS.

187 Current asthma

188 MACS defined current asthma as one or more episodes of asthma and/or the use of

189 any asthma medications in the last 12 months. LISAplus defined current asthma as

190 doctor diagnosis of asthma at the age of 10 years or intake of asthma medication

191 during the past 12 months.

192 Current Allergic rhinitis

193 MACS defined current allergic rhinitis as one or more episodes of allergic rhinitis

194 and/or the use of any treatment for allergic rhinitis in the last 12 months. LISAplus 
195 defined allergic rhinitis as doctor diagnosis of seasonal and/or perennial rhinitis at the 196 age of 10 years.

\section{Confounder definitions}

199 Eczema by the age at which the sensitization was assessed:

200 Defined as doctor diagnosis or treatment of rash with topical steroid (excluding nappy 201 or scalp areas) by the age of SPT in MACS and as doctor diagnosis of eczema in the 202 past 6 months and/or rash in the past 6 months asked at the age of 2 years in

203 LISAplus.

204 Wheeze by the age at which the sensitization was assessed:

205 Defined if the response to the following question was $>5$ days "How many days of 206 cough and/or chest rattle and/or wheeze has your child had in the past 4 weeks?" in 207 MACS and according to the response to the following question "In the past 6 months, 208 has your child had whistling or wheezy sound of breathing in the chest?" asked during 209 the follow-up at age 2 years in LISAplus.

\section{Statistical Analysis}

212 Logistic regression models were constructed with asthma or allergic rhinitis as

213 dependent variables and food and/or aeroallergen sensitization at 6, 12 or 24 months

214 (in MACS) or at 24 months (in LISAplus) as independent variables. The predictive

215 evaluation of sensitization was based on four groups: (1) no sensitization, (2) food

216 sensitization only, (3) aeroallergen sensitization only and (4) sensitization to both

217 food and aeroallergen. In MACS, the associations were evaluated at each time point

218 separately, irrespective of previous status of sensitization. All models were adjusted

219 for sex, maternal smoking during pregnancy, parental level of education, exclusive

220 breastfeeding for at least 4 months (30) and eczema by the age of sensitization. The

221 association between atopic sensitization and asthma was further adjusted for wheeze

222 by the age at the assessment of sensitization.

223 Additional adjustment for formula allocation was performed in MACS analyses and

224 for study center and family history of allergy (whether mother, father or a sibling ever

225 had asthma, eczema or hay fever; asked at birth) in LISAplus. An interaction between

226 allergic sensitization and family history of allergy was tested in LISAplus.

227 Results are presented as crude and adjusted Odd Ratios (OR) and 95\% confidence

228 intervals. All statistical tests were two sided with a $p$ value of $<0.05$ considered as 

statistically significant. STATA 13 (StataCorp, College Station TX) was used in all analyses in MACS and R version 3.1.0 was used for all analyses in LISAplus (31).

\section{Results}

Characteristics of the study populations

$$
\text { The characteristics for analyzed MACS participants are presented in Table 1. At all }
$$
time points tested in MACS, egg white was the commonest food allergen (13\%, $18 \%$ and $15 \%$ at 6, 12 and 24 months, respectively), while house dust mite was the most prevalent aeroallergen $(5 \%, 12 \%$ and $23 \%$ at 6,12 and 24 months, respectively._With the exception of maternal education and paternal smoking, MACS participants who did not attend the 12 year follow-up were similar on all demographic characteristics and early life sensitization compared to those who did attend (see Table E1 in the

\section{Online Repository).}

The characteristics for participants analyzed from LISAplus (630, 292, 138 and 120 participants from Munich, Leipzig, Bad Honnef and Wesel, respectively) are presented in Table 1. At 24 months in LISAplus, the major food allergens were egg white and milk protein (5\% each) and the major aeroallergen was house dust mite (3\%). Apart from parental education, maternal smoking, number of older siblings, a sibling ever having asthma and parental history of food allergy or hay fever, LISAplus participants who did not attend the 10 year follow-up were similar on other demographic characteristics and sensitization at 24 months compared to those who did attend (see Table E1 in the Online Repository).

\section{Atopic sensitization and allergic airway diseases}

\section{Food sensitization and asthma and allergic rhinitis}

In MACS, infants who were sensitized to food allergens without concurrent aeroallergen sensitization at 12 months had an increased risk of current asthma and allergic rhinitis compared to non-sensitized infants. While there were similar trends at 6 and 24 months, these were not significant (Tables 2\&3). Additionally, children who had co-sensitization to both food and aeroallergen at 6, 12 or 24 months had increased risk of current asthma and allergic rhinitis. These associations, at all three time points, appeared stronger than sensitization to food without sensitization to aeroallergen (Tables 2\&3). 
262 In LISAplus, children who were sensitized to food without concurrent aeroallergen at

26324 months had an increased risk of current asthma and allergic rhinitis compared to

264 non-sensitized children (Tables 2\&3). Similar to MACS, the strongest effect on

265 asthma and allergic rhinitis risk was observed in children who had co-sensitization to

266 food and aeroallergen at 24 months (Tables 2\&3).

268 Aeroallergen only sensitization and asthma and allergic rhinitis

269 In MACS, sensitization to aeroallergen without food at 12 months, but not at 6

270 months was associated with increased risk of current asthma and allergic rhinitis

271 (Tables 2\&3). Moreover, children who had aeroallergen without food sensitization at

27224 months in both cohorts had increased risks of current asthma and allergic rhinitis

273 and these associations were weaker than sensitization to both food and aeroallergen

274 (Tables 2\&3).

276 None of the above associations were modified by family history of allergy in

277 LISAplus study (i.e. $p$ value of interaction was $>0.1$ ).

\section{Discussion}

280 Our study has shown that food sensitization in the first two years, independent of

281 early life eczema and wheeze, predicts asthma and allergic rhinitis in later childhood.

282 Additionally, co-sensitization to both food and aeroallergen was the strongest

283 predictor at any time point tested. Our findings were mostly consistent across two

284 cohorts, where data were available, with different populations in relation to co-

285 sensitization to food and aeroallergen and sensitization to aeroallergen only. The

286 MACS is an Australian cohort of children with a family history of allergic diseases

287 while LISAplus is a German population-based cohort. Interestingly, our findings were

288 similar among those with and without a family history within the LISAplus study.

289 Earlier studies have established the role of aeroallergen sensitization on development

290 of allergic airway diseases (13-16). We established that food sensitization itself was

291 related to subsequent increased risk of asthma and allergic rhinitis. The current

292 analysis compared the effect of different mutually exclusive groups of atopic

293 sensitization on asthma and allergic rhinitis in later childhood allowing us to draw

294 stronger conclusions on the different patterns of sensitization. Few studies have

295 investigated the role of early life food sensitization on development of asthma and 
296 allergic rhinitis beyond the age of 7 years (22-24). Bekkers and colleagues (24)

297 showed that egg, but not cow's milk sensitization at 12 months was associated with

298 increased risk of asthma up to the age of 10 years. However, whether the observed

299 effect was due to sensitization to food alone without concurrent aeroallergen was not

300 assessed. Additionally, potential confounding by early life eczema and/or wheeze was

301 not considered in this analysis.

302 Our study showed that food without aeroallergen sensitization at 24 months was 303 associated with increased risks of asthma and allergic rhinitis in the LISAplus study 304 but not in MACS. This appears to be due, at least in part, to only a small number of 305 MACS participants only having food sensitization at 24 months compared to the 306 LISAplus study. In contrast, findings related to aeroallergen without food 307 sensitization and co-sensitization to foods and aeroallergen were consistent across 308 both cohorts.

309 We found also that children co-sensitized to common foods and aeroallergen at any 310 time point tested had a markedly higher risk of developing respiratory allergic

311 diseases than sensitization to food or aeroallergen alone when compared to non-

312 sensitized children. Few longitudinal studies have assessed the association between

313 co-sensitization to food and aeroallergen and development of atopic diseases in

314 childhood. A German study by Illi et al. (32) showed that concurrent sensitization to 315 food and aeroallergen was the strongest predictor for asthma up to school age. In an 316 Australian study, Garden et al. (23) found that mixed food and inhalant sensitization 317 phenotype had the strongest associations with allergic disease at the age of 8 years, 318 and particularly with asthma. However, co-manifestation of early life wheeze and/or 319 eczema was not been taken into account in the analysis and there was a shorter period 320 of follow-up.

321 Eczema in early life is commonly associated with high levels of food specific IgE 322 (33), and has been associated with increased risk of later asthma and allergic rhinitis 323 (9). Similarly, wheeze has been related to food specific $\operatorname{IgE}$ (34). When early life 324 eczema and/or wheezing, reported at the same age of testing for atopic sensitization, 325 were considered in our analysis, the associations between atopic sensitization and 326 allergic outcomes did not change significantly. This suggests that the role of early life 327 sensitization on later childhood allergic airway diseases is independent of eczema 328 and/or wheezing. 
329 The strengths of this study are that we have analyzed longitudinal data from two 330 independent cohorts with long periods of follow-up (extending from infancy to later

331 childhood), the relatively large sample sizes and early objective assessment of

332 sensitization to common allergens. These cohorts were from two different regions of

333 the world, but both were high income countries with high prevalences of food

334 sensitization (35) and allergic diseases (36-38). It is often assumed that results from a

335 high-risk cohort may not be applicable to the general population, but interestingly our 336 results were similar across the two cohorts. Also, family history of allergic diseases

337 did not modify our associations in the population-based cohort suggesting that family

338 history may not be a major modifier of risk and that other factors should be

339 considered, for example environmental exposures.

340 This study has a number of limitations. Our analyses were based on longitudinal data

341 and loss to follow-up needs to be considered when interpreting the results as it could

342 be a potential source of bias. However, these studies achieved a 57\% attendance at the

34310 year follow-up in LISAplus and 59\% attendance at the 12 year follow-up in

344 MACS. In addition, apart from parental education and paternal smoking, there were

345 no significant demographic and/or early sensitization differences between children

346 who did and did not attend in either cohort. Although the reported paternal history of

347 food allergy and hay fever was higher in those who attended the 10 years follow-up in

348 LISAplus, this was unlikely to bias findings. Another possible limitation is that the

349 definitions of asthma and allergic rhinitis were based on questionnaire data. However,

350 these definitions have been commonly used in epidemiological studies $(20,39)$. The

351 findings of this study could have been strengthened if participants were examined for

352 objective evidence of asthma and allergic rhinitis. Moreover, we were unable to

353 establish the associations between specific allergen sensitization and subsequent

354 development of asthma and allergic rhinitis due to limited statistical power.

355 Previous studies have observed that positive SPT reactions are likely to be smaller in

356 infants and children younger than two years (40) presumably because of a relative

357 lack of allergen-specific IgE and skin reactivity (41). Therefore, in the MACS cohort,

358 a $2 \mathrm{~mm}$ cut-off point was used to define positive skin prick test reactions at 6, 12 and

35924 months (42).

360 We acknowledge that food sensitization was assessed by SPT in MACS and by serum

361 IgE in LISAplus. These two methods are commonly used to evaluate sensitization in

362 epidemiological studies (43). Many studies have assessed sensitization as a predictor 
363 of allergic diseases, either by $\operatorname{SPT}(12,22,23)$ or s-IgE $(12,20,24,44)$. A recent

364 study by Ro et al. (45) showed that the predictive value of SPT and s-IgE performed

365 at 2 years of age was generally comparable in predicting allergic diseases in later

366 childhood.

367 In conclusion, assessment of food sensitization in infants provides valuable

368 information on the risk of later childhood asthma and allergic rhinitis. Additionally,

369 we provide evidence for the role of early life food sensitization with or without co-

370 sensitization to aeroallergen, independent of early life eczema, on the atopic march.

371 Developing interventions that prevent early life food sensitization, such as food

372 allergen avoidance or dietary modification, may reduce the likelihood of atopic march

373 to asthma and allergic rhinitis occurring.

374

375 Acknowledgment

376 For MACS study, we thank Dr John Thorburn, FRACP, for assistance in patient

377 recruitment and administrative assistance and the Mercy Maternity Hospital

378 Department of Obstetrics for participant recruitment, and Dr Cliff Hosking for study

379 leadership up to the 12 year follow-up. We thank Anne Balloch for assistance with

380 data management. Most importantly, we thank all of the MACS children and parents

381 for their participation and ongoing support for this study.

383 The LISAplus Study Group consists of the following: Helmholtz Zentrum München,

384 German Research Center for Environmental Health, Institute of Epidemiology,

385 Munich (Heinrich J, Wichmann HE, Sausenthaler S, Chen CM, Schnappinger M);

386 Department of Pediatrics, Municipal Hospital "St.Georg”, Leipzig (Borte M, Diez U),

387 Marien-Hospital Wesel, Department of Pediatrics, Wesel (von Berg A, Beckmann C,

388 Groß I); Pediatric Practice, Bad Honnef (Schaaf B); Helmholtz Centre for

389 Environmental Research - UFZ, Department of Environmental Immunology/Core

390 Facility Studies, Leipzig (Lehmann I, Bauer M, Gräbsch C, Röder S, Schilde M);

391 University of Leipzig, Institute of Hygiene and Environmental Medicine, Leipzig

392 (Herbarth O, Dick C, Magnus J); IUF - Leibniz Research Institute for Environmental

393 Medicine, Düsseldorf (Krämer U, Link E, Cramer C); Technical University Munich,

394 Department of Pediatrics, Munich (Bauer CP, Hoffmann U); ZAUM - Center for

395 Allergy and Environment, Technical University, Munich (Behrendt H, Grosch J,

396 Martin F).

This article is protected by copyright. All rights reserved 


\section{Declaration of all source of funding}

399 SAA has scholarship from King Saud University, Riyadh-Saudi Arabia represented 400 by Saudi Arabian Cultural Mission in Canberra-Australia; CJL is funded by The 401 National Health and Medical Research Council of Australia (NHMRC); AJL and 402 SCD are funded by the NHMRC; KJA is funded by the Charles and Sylvia Viertel 403 Charitable Foundation. MJA holds investigator initiated research grants from Pfizer 404 and Boehringer Ingelheim. He received support to attend the ERS Congress from 405 Boehringer Ingelheim and attended a Respiratory Symposium funded by 406 Novartis. He has been paid honoraria by Astra Zeneca and Novartis. These do not 407 constitute substantial sources of income. BE, MS, AB and JH have no personal 408 funding relationships to declare.

409 Initial funding for the MACS in the first six years of the study was from Nestec (a 410 subsidiary of Nestlé Australia.) The 12 year follow-up was supported by the Asthma 411 Foundation of Victoria. The funding bodies had no role in the study design, 412 collection, analysis or interpretation of data, nor in writing this report or the decision 413 to publish. The results, conclusions and opinions reported in the manuscript are those 414 of the authors and are independent from the funding sources.

415 The LISAplus study was mainly supported by grants from the Federal Ministry for 416 Education, Science, Research and Technology and in addition from Helmholtz 417 Zentrum Munich (former GSF), Helmholtz Centre for Environmental Research 418 UFZ, Leipzig, Research Institute at Marien-Hospital Wesel, Pediatric Practice, Bad 419 Honnef for the first 2 years. The 4 year, 6 year, and 10 year follow-up examinations 420 of the LISAplus study were covered from the respective budgets of the involved 421 partners (Helmholtz Zentrum Munich (former GSF), Helmholtz Centre for

422 Environmental Research - UFZ, Leipzig, Research Institute at Marien-Hospital 423 Wesel, Pediatric Practice, Bad Honnef, IUF - Leibniz-Research Institute for 424 Environmental Medicine at the University of Düsseldorf) and in addition by a grant 425 from the Federal Ministry for Environment (IUF Düsseldorf, FKZ 20462296).

\section{Conflict of interest}

428 No conflict of interest to declare. 
432 1. Asher MI, Montefort S, Björkstén B, Lai CKW, Strachan DP, Weiland SK, et al.

433 Worldwide time trends in the prevalence of symptoms of asthma, allergic rhinoconjunctivitis,

434 and eczema in childhood: ISAAC Phases One and Three repeat multicountry cross-sectional

435 surveys. Lancet. 2006;368(9537):733-43.

436 2. Prescott S, Allen KJ. Food allergy: riding the second wave of the allergy epidemic.

437 Pediatric Allergy and Immunology. 2011;22(2):155-60.

438 3. Nwaru B, Hickstein L, Panesar S, Muraro A, Werfel T, Cardona V, et al. The

439 epidemiology of food allergy in Europe: a systematic review and meta - analysis. Allergy.

$440 \quad 2014 ; 69(1): 62-75$.

4414 Lewis-Jones S. Quality of life and childhood atopic dermatitis: the misery of living

442 with childhood eczema. International Journal Of Clinical Practice. 2006;60(8):984-92.

443 5. Meltzer EO. Quality of life in adults and children with allergic rhinitis. Journal of

444 Allergy and Clinical Immunology. 2001;108(1, Supplement):S45-S53.

445 6. Guilbert T, Guilbert C, Garris P, Jhingran M, Bonafede K, Tomaszewski T, et al.

446 Asthma That Is Not Well-Controlled Is Associated with Increased Healthcare Utilization and

447 Decreased Quality of Life. The Journal of asthma. 2011;48(2):126-32.

448 7. Zheng T, Yu J, Oh MH, Zhu Z. The atopic march: progression from atopic dermatitis

449 to allergic rhinitis and asthma. Allergy, asthma \& immunology research. 2011;3(2):67-73.

450 8. Das RR. Food Allergy, Atopic March and United Airway Diseases. 2012.

451 9. Lowe A, Hosking C, Bennett C, Carlin J, Abramson M, Hill D, et al. Skin prick test

452 can identify eczematous infants at risk of asthma and allergic rhinitis. Clinical \&

453 Experimental Allergy. 2007;37(11):1624-31.

454 10. Flohr C, Johansson S, Wahlgren C-F, Williams H. How atopic is atopic dermatitis?

455 Journal of Allergy and Clinical Immunology. 2004;114(1):150-8.

456 11. Ker J, Hartert TV. The atopic march: what's the evidence? Annals of Allergy, Asthma 457 \& Immunology. 2009;103(4):282-9.

458 12. Kjaer H, Eller E, Andersen K, Host A, Bindslev Jensen C, HÃ st A. The association 459 between early sensitization patterns and subsequent allergic disease. The DARC birth cohort 460 study. Pediatric Allergy and Immunology. 2009;20(8):726-34.

461 13. Lau S, Nickel R, Niggemann B, Grüber C, Sommerfeld C, Illi S, et al. The

462 development of childhood asthma: lessons from the German Multicentre Allergy Study

463 (MAS). Paediatric respiratory reviews. 2002;3(3):265-72.

464 14. Codispoti CD, Levin L, LeMasters GK, Ryan P, Reponen T, Villareal M, et al.

465 Breast-feeding, aeroallergen sensitization, and environmental exposures during infancy are 
determinants of childhood allergic rhinitis. Journal of Allergy and Clinical Immunology. 2010;125(5):1054-60. e1.

468 15. Lodge CJ, Lowe AJ, Gurrin LC, Hill DJ, Hosking CS, Khalafzai RU, et al. House

469 dust mite sensitization in toddlers predicts current wheeze at age 12 years. Journal of Allergy and Clinical Immunology. 2011;128(4):782-8. e9.

471 16. Schäfer T, Wölke G, Ring J, Wichmann HE, Heinrich J. Allergic sensitization to cat

472 in childhood as major predictor of incident respiratory allergy in young adults. Allergy.

$473 \quad 2007 ; 62(11): 1282-7$.

474 17. Weinberg EG. The atopic march. Curr Allergy Clin Immunol. 2005;18(1):4-5.

475 18. Nickel R, Lau S, Niggemann B, Grüber C, Von Mutius E, Illi S, et al. Messages from 476 the German Multicentre Allergy Study. Pediatric Allergy and Immunology. 2002;13:7-10.

477 19. Schnabel E, Sausenthaler S, Schaaf B, Schäfer T, Lehmann I, Behrendt H, et al.

478 Prospective association between food sensitization and food allergy: results of the LISA birth 479 cohort study. Clinical \& Experimental Allergy. 2010;40(3):450-7.

480 20. Brockow I, Zutavern A, Hoffmann U, Grubl A, Von Berg A, Koletzko S, et al. 3

481 Early Allergic Sensitizations and Their Relevance to Atopic Diseases in Children Aged 6

482 Years: Results of the GINI Study. Journal of investigational allergology \& clinical

483 immunology. 2009;19(3):180.

484 21. Burr M, Merrett T, Dunstan F, Maguire M. The development of allergy in high - risk 485 children. Clinical \& Experimental Allergy. 1997;27(11):1247-53.

486 22. Rhodes HL, Sporik R, Thomas P, Holgate ST, Cogswell JJ. Early life risk factors for 487 adult asthma: A birth cohort study of subjects at risk. Journal of Allergy and Clinical

488 Immunology. 2001;108(5):720-5.

489 23. Garden FL, Simpson JM, Marks GB. Atopy phenotypes in the Childhood Asthma

490 Prevention Study (CAPS) cohort and the relationship with allergic disease. Clinical \&

491 Experimental Allergy. 2013;43(6):633-41.

492 24. Bekkers MB, Aalberse RC, Gehring U, Kerkhof M, Koppelman GH, de Jongste JC, 493 et al. Hen's egg, not cow's milk, sensitization in infancy is associated with asthma: 10-year 494 follow-up of the PIAMA birth cohort. Journal of Allergy and Clinical Immunology. 495 2013;132(6):1427-8.

496 25. Howard G, Howard VJ. Observational Epidemiology within Randomized Clinical

497 Trials: Getting a lot for (almost) nothing. Progress in cardiovascular diseases.

498 2012;54(4):367-71.

499 26. Lowe AJ, Hosking CS, Bennett CM, Allen KJ, Axelrad C, Carlin JB, et al. Effect of a 500 partially hydrolyzed whey infant formula at weaning on risk of allergic disease in high-risk 
501 children: a randomized controlled trial. Journal of Allergy and Clinical Immunology.

502 2011;128(2):360-5. e4.

503 27. Heinrich J, Bolte G, Hölscher B, Douwes J, Lehmann I, Fahlbusch B, et al. Allergens

504 and endotoxin on mothers' mattresses and total immunoglobulin $\mathrm{E}$ in cord blood of neonates.

505 European Respiratory Journal. 2002;20(3):617-23.

506 28. Heinzerling L, Mari A, Bergmann K-C, Bresciani M, Burbach G, Darsow U, et al.

507 The skin prick test-European standards. Clinical and translational allergy. 2013;3(1):1-10.

508 29. Rhodes HL, Thomas P, Sporik R, Holgate ST, Cogswell JJ. A birth cohort study of

509 subjects at risk of atopy: Twenty-two-year follow-up of wheeze and atopic status. American

510 journal of respiratory and critical care medicine. 2002;165(2):176-80.

511 30. Fleischer DM, Spergel JM, Assa'ad AH, Pongracic JA. Primary prevention of allergic

512 disease through nutritional interventions. The Journal of Allergy and Clinical Immunology: In

513 Practice. 2013;1(1):29-36.

514 31. Statistical Package R. R: A language and environment for statistical computing.

515 Vienna, Austria: R Foundation for Statistical Computing. 2009.

516 32. Illi S, von Mutius E, Lau S, Nickel R, Niggemann B, Sommerfeld C, et al. The

517 pattern of atopic sensitization is associated with the development of asthma in childhood.

518 Journal of Allergy and Clinical Immunology. 2001;108(5):709-14.

519 33. Hill DJ, Sporik R, Thorburn J, Hosking CS. The association of atopic dermatitis in

520 infancy with immunoglobulin E food sensitization. The Journal of pediatrics.

$5212000 ; 137(4): 475-9$.

522 34. Wang J, Visness CM, Sampson HA. Food allergen sensitization in inner-city children

523 with asthma. Journal of Allergy and Clinical Immunology. 2005;115(5):1076-80.

524 35. Burney P, Summers C, Chinn S, Hooper R, Van Ree R, Lidholm J. Prevalence and

525 distribution of sensitization to foods in the European Community Respiratory Health Survey:

526 a EuroPrevall analysis. Allergy. 2010;65(9):1182-8.

527 36. Stock S, Redaelli M, Luengen M, Wendland G, Civello D, Lauterbach K. Asthma: 528 prevalence and cost of illness. European Respiratory Journal. 2005;25(1):47-53.

529 37. Maziak W, Behrens T, Brasky T, Duhme H, Rzehak P, Weiland S, et al. Are asthma

530 and allergies in children and adolescents increasing? Results from ISAAC phase I and phase

531 III surveys in Münster, Germany. Allergy. 2003;58(7):572-9.

532 38. Pearce N, Ait-Khaled N, Beasley R, Mallol J, Keil U, Mitchell E, et al. Worldwide

533 trends in the prevalence of asthma symptoms: phase III of the International Study of Asthma

534 and Allergies in Childhood (ISAAC) 2007.

535 39. Havstad S, Johnson CC, Kim H, Levin AM, Zoratti EM, Joseph CL, et al. Atopic

536 phenotypes identified with latent class analyses at age 2 years. Journal of Allergy and Clinical

537 Immunology. 2014. 
539 diagnostic testing: an updated practice parameter. Annals of Allergy, Asthma \& Immunology.

540 2008;100(3):S1-S148.

541 41. Ménardo JL, Bousquet J, Rodière M, Astruc J, Michel F-B. Skin test reactivity in

542 infancy. Journal of Allergy and Clinical Immunology. 1985;75(6):646-51.

543 42. Alduraywish SA, Lodge CJ, Vicendese D, Lowe AJ, Erbas B, Matheson MC, et al.

544 Sensitization to milk, egg and peanut from birth to 18 years: A longitudinal study of a cohort

545 at risk of allergic disease. Pediatric Allergy and Immunology. 2016;27(1):83-91.

546 43. Bousquet PJ, Bousquet R, Hooper M, Kogevinas D, Jarvis P, Burney. Number of

547 allergens to be tested to assess allergenic sensitization in epidemiologic studies: results of the

548 European Community Respiratory Health Survey I. Clinical and experimental allergy.

549 2007;37(5):780-7.

550 44. Kulig M, Bergmann R, Tacke U, Wahn U, Guggenmoos - Holzmann I. Long -

551 lasting sensitization to food during the first two years precedes allergic airway disease.

552 Pediatric Allergy and Immunology. 1998;9(2):61-7.

553 45. Rø AD, Simpson MR, Storrø O, Johnsen R, Videm V, Øien T. The predictive value

554 of allergen skin prick tests and IgE tests at pre - school age: The PACT study. Pediatric

555 Allergy and Immunology. 2014;25(7):691-8.

556

557

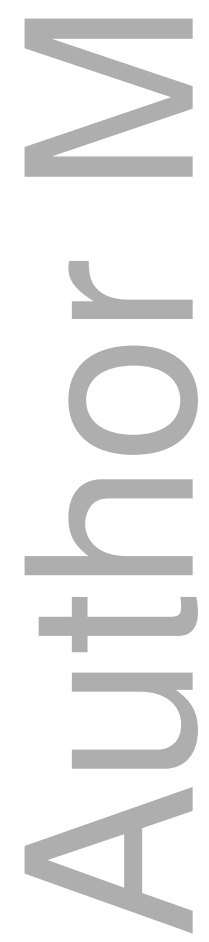


Table 1: Characteristics of participants analyzed from the MACS and LISAplus studies

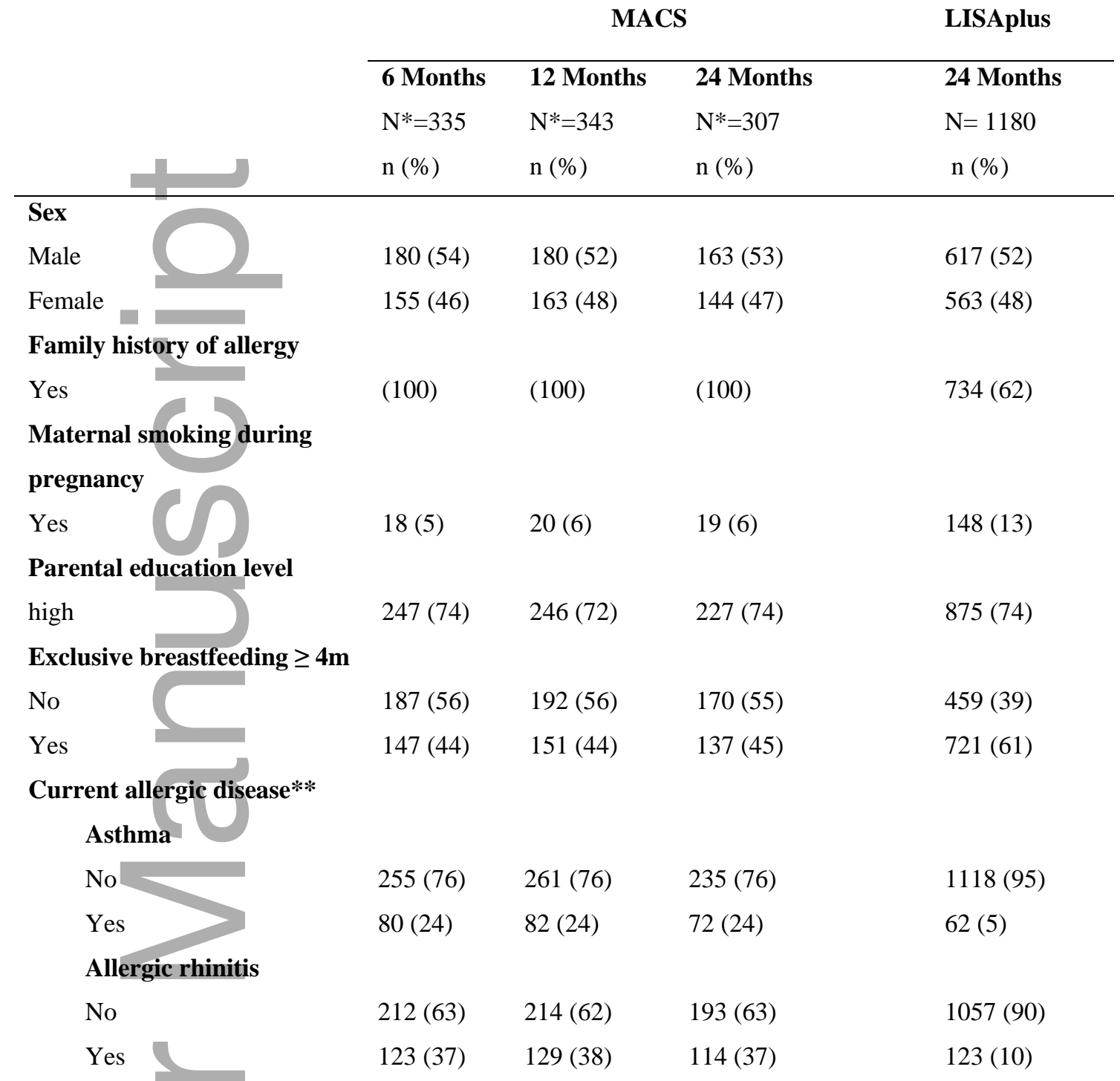

\footnotetext{
$* \mathrm{~N}$ represents the number of participants who had available data on both sensitization and allergic diseases (asthma and allergic rhinitis).

**At 10Y in LISAplus and 12Y in MACS.
}

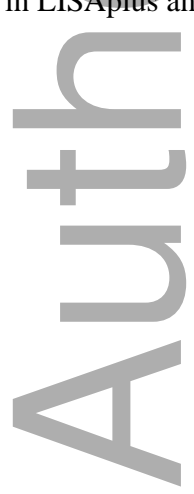


Table 2: The association between food only, aeroallergen only and both food and aeroallergen sensitization and asthma in MACS and LISAplus.

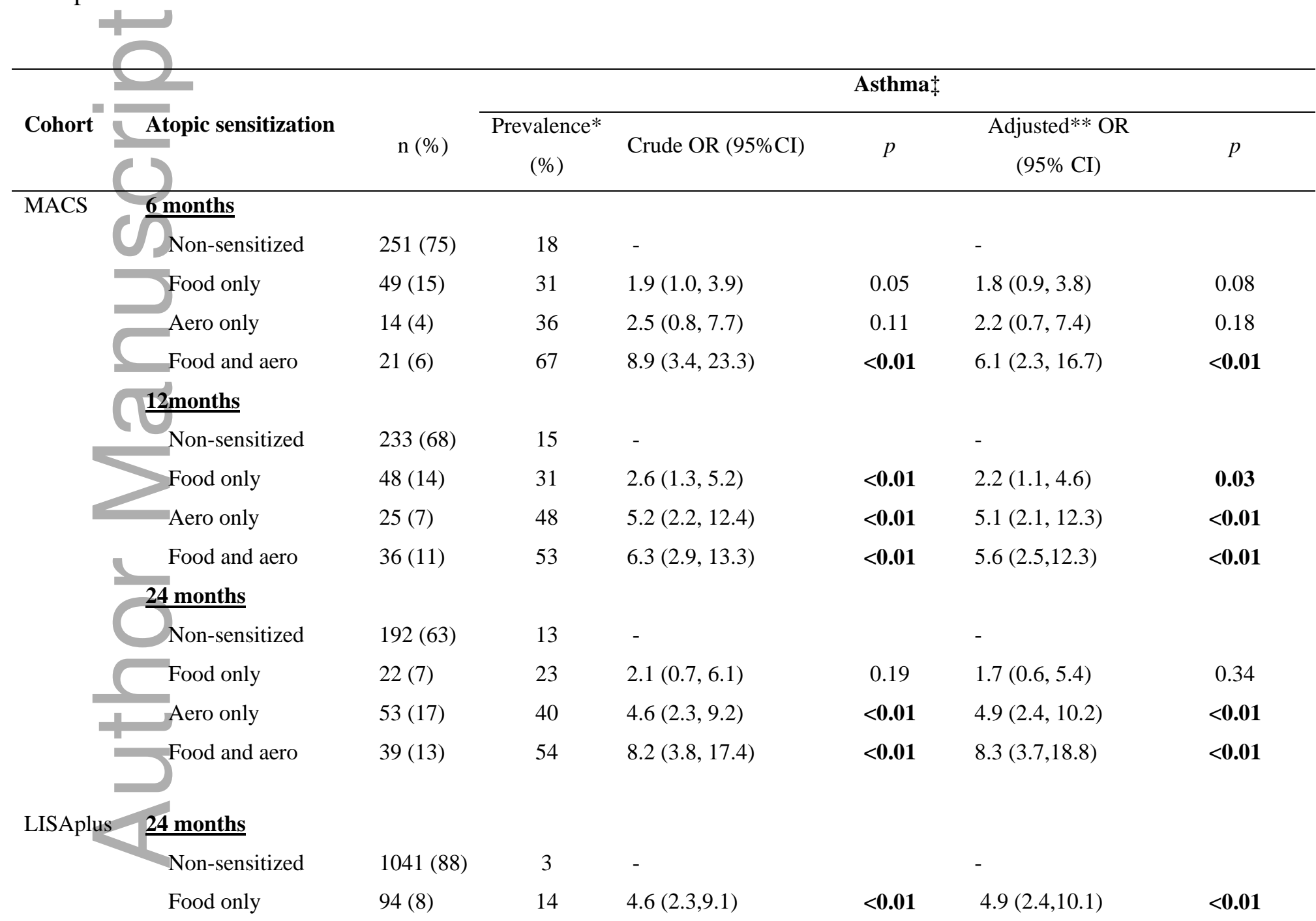

This article is protected by copyright. All rights reserved 


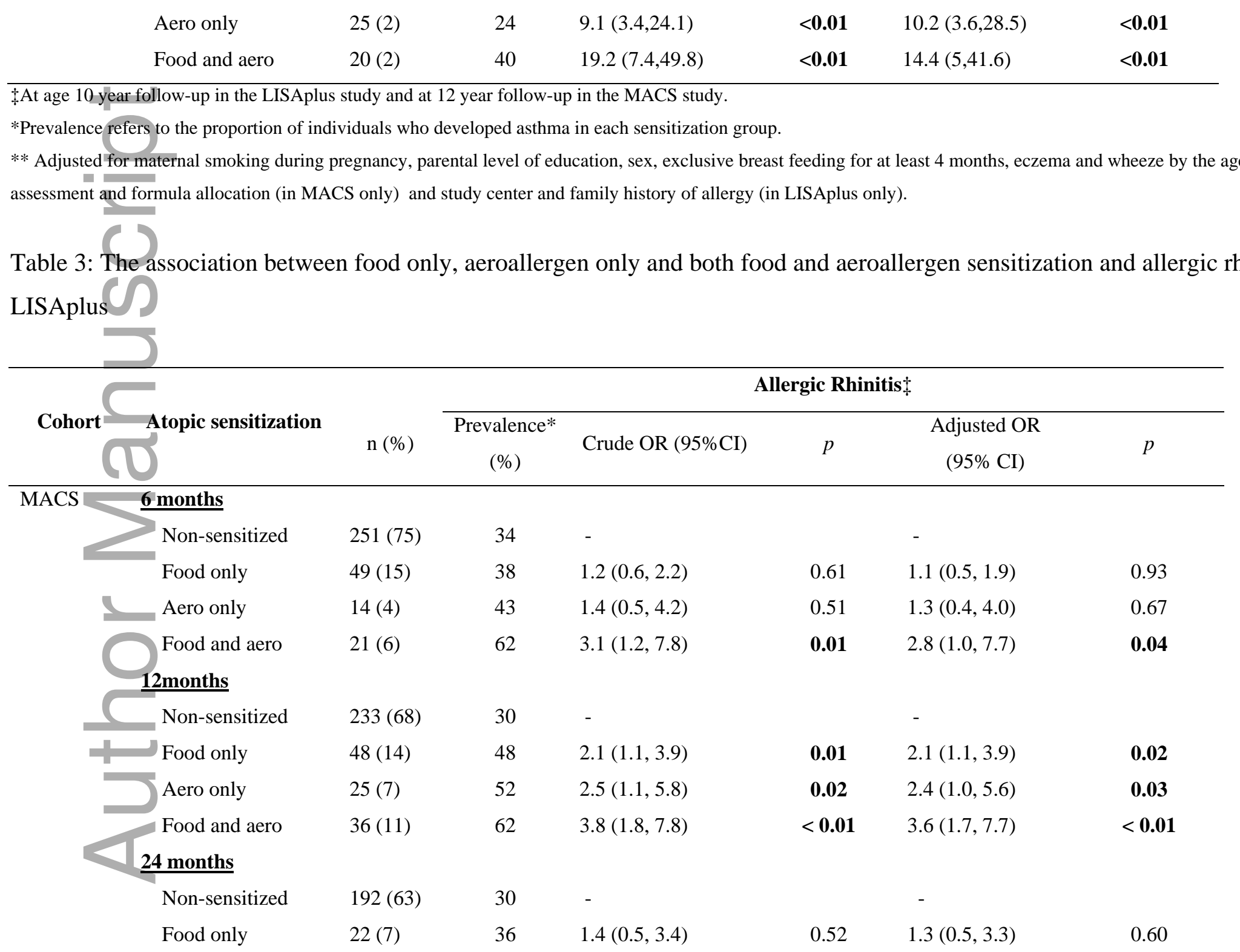

This article is protected by copyright. All rights reserved 


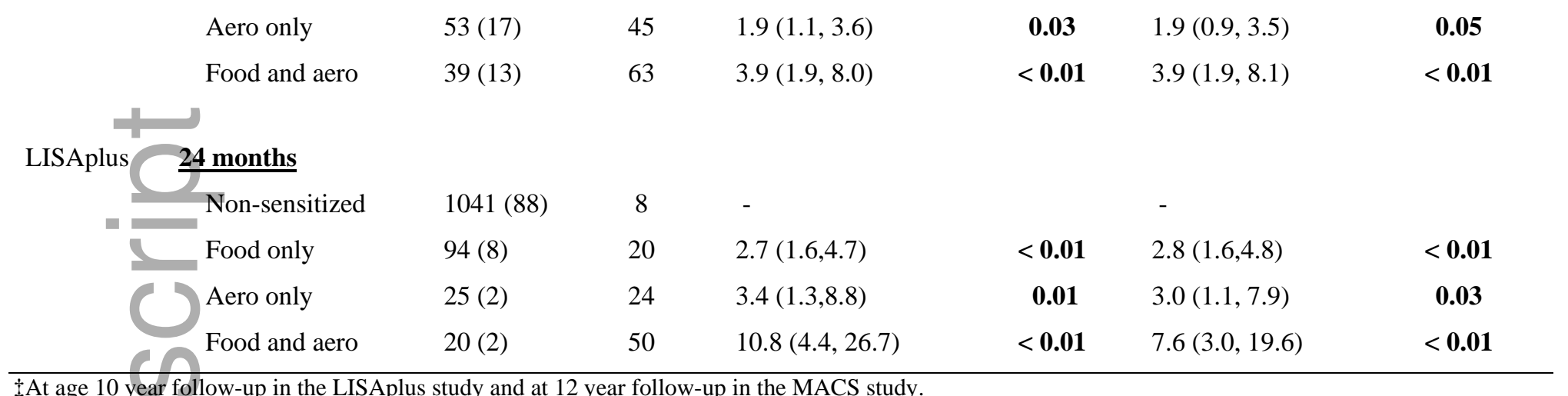

$\$$ At age 10 year follow-up in the LISAplus study and at 12 year follow-up in the MACS study.

*Prevalence refers to the proportion of individuals who developed allergic rhinitis in each sensitization group.

** Adjusted for maternal smoking during pregnancy, parental level of education, sex, exclusive breast feeding for at least 4 months, eczema by the age of sensitization assessment and formula allocation ( in MACS only) and study center and family history of allergy ( in LISAplus only)

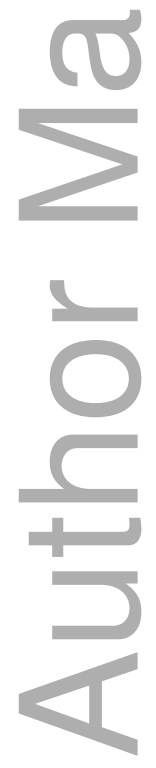

This article is protected by copyright. All rights reserved 


\section{University Library}

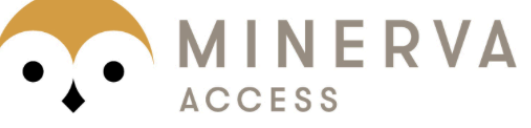

A gateway to Melbourne's research publications

Minerva Access is the Institutional Repository of The University of Melbourne

\section{Author/s:}

Alduraywish, SA;Standl, M;Lodge, CJ;Abramson, MJ;Allen, KJ;Erbas, B;von Berg,

A;Heinrich, J;Lowe, AJ;Dharmage, SC

Title:

Is there a march from early food sensitization to later childhood allergic airway disease? Results from two prospective birth cohort studies

Date:

2017-02-01

Citation:

Alduraywish, S. A., Standl, M., Lodge, C. J., Abramson, M. J., Allen, K. J., Erbas, B., von Berg, A., Heinrich, J., Lowe, A. J. \& Dharmage, S. C. (2017). Is there a march from early food sensitization to later childhood allergic airway disease? Results from two prospective birth cohort studies. PEDIATRIC ALLERGY AND IMMUNOLOGY, 28 (1), pp.30-37. https:// doi.org/10.1111/pai.12651.

Persistent Link:

http://hdl.handle.net/11343/291834 